\title{
TOWARDS CONTROLLED PEELING AS A PROCESS TO RECOVER REUSABLE ORGANOSHEET LAMINAE
}

\author{
$\underline{\text { S. Arnold-Keifer }}{ }^{*}$, M. Imbert ${ }^{1}$, N. Groß ${ }^{1}$, M. May ${ }^{1}$ \\ ${ }^{1}$ Fraunhofer Institute for High-Speed-Dynamics, Ernst Mach Institute, EMI, \\ Ernst Zermelo Strasse 4, 79104 Freiburg, Germany \\ * sonja.arnold-keifer@emi.fraunhofer.de
}

Organosheets are multi-layer thermoplastic composites rapidly gaining interest in the automotive industry due to their ability to replace metallic sheets in lightweight applications. However, the current recycling approach for these materials mainly consists in strongly damaging mechanical processing such as grinding or shredding [1]. This leads to the loss of fibre length and alignment, which were causing the high mechanical properties of the original material. Therefore, the recycled material cannot be reused for the same application. However, the thermoplastic composites feature the major advantage of being reprocessable which would for example enable to reuse laminae (in polymer embedded unitary reinforcement layers) for the same application or as reinforcing patches if they could be recovered without significant damage from the multi-layer structure.

In this context, the motivation of the presented work is to use a fracture mechanics framework to design a new high-quality recycling process based on the controlled delamination of organosheet laminae. The focus of this work is the use of peeling to recover minimally damaged laminae. To reach this goal, an analytical model describing the peeling process, accounting for the cohesive zone ahead of the crack tip as well as for the loading state and damage in the peeling arm is developed.

The model is used to investigate the relative influence of the material properties and applied loads on crack propagation. For various sets of material properties, various types of loads as axial loads or normal loads located at various positions on the peel arm are tested. Crack propagation and potential damages induced in the peel arm by the applied loads are outputs of the sensitivity analyses. These analyses enable identifying quantitatively the loading conditions ensuring separation with a minimal damage of the recovered lamina.

Finally, the model is fed with experimental data (mechanical properties, fracture toughness, etc) obtained on organo-sheet materials used commonly in the industry and validation tests are conducted.

The use of failure mechanics to develop innovative recovering techniques open the way for novel high-quality recycling processes for composites ensuring both high mechanical and economical values of the recycled material.

\section{References}

[1] Oliveux, G., Dandy, L. O., \& Leeke, G. A. (2015). Current status of recycling of fibre reinforced polymers: Review of technologies, reuse and resulting properties. Progress in Materials Science, 72, 61-99. 